\title{
SKELETONS OF HYPER MV-ALGEBRAS
}

\author{
Min Su KANG
}

\begin{abstract}
Several types of skeletons in a hyper MV-algebra are introduced, and their relations are investigated.

\section{Introduction}

In order to provide an algebraic proof of completeness theorem of infinite valued Lukasewicz propositional calculus, Chang [1] introduced the notion of MV-algebras. The hyper structure theory was introduced by Marty [5] at 8th congress of Scandinavian Mathematicians in 1934. Since then many researchers have worked in these areas. Recently in [3], Ghorbani et al. applied the hyper structure to MV-algebras and introduced the concept of a hyper MV-algebra which is a generalization of an MV-algebra and investigated some related results. Torkzadeh and Ahadpanah [6] discussed hyper MV-ideals in hyper MV-algebras. Jun et al. [4] studied hyper MV-deductive systems in hyper MV-algebras. In this paper, we introduce several types of skeletons in hyper MV-algebras, and investigate their relations.
\end{abstract}

\section{Preliminaries}

Definition 2.1. [2] A hyper $M V$-algebra is a nonempty set $M$ endowed with a hyper operation " $\oplus$ ", a unary operation " $*$ " and a constant " 0 " satisfying the following axioms:

(a1) $x \oplus(y \oplus z)=(x \oplus y) \oplus z$,

(a2) $x \oplus y=y \oplus x$

(a3) $\left(x^{*}\right)^{*}=x$

(a4) $\left(x^{*} \oplus y\right)^{*} \oplus y=\left(y^{*} \oplus x\right)^{*} \oplus x$,

(a5) $0^{*} \in x \oplus 0^{*}$,

Received December 29, 2009. Accepted March 8, 2010.

Keywords: Hyper MV-algebra, (weak) Hyper MV-skeleton, $(\subseteq, \in)$-skeleton, ( $\epsilon)$-skeleton, $\left(\ll^{*}, \in\right)$-skeleton, (strong) $\left(\subseteq^{*}, \in\right)$-skeleton

2000 Mathematics Subject Classification. 06F35, 06D35, 03G25. 
(a6) $0^{*} \in x \oplus x^{*}$,

(a7) $x \ll y, y \ll x \Rightarrow x=y$

for all $x, y, z \in M$, where $x \ll y$ is defined by $0^{*} \in x^{*} \oplus y$.

For every subsets $A$ and $B$ of $M$, we define

$$
\begin{gathered}
A \ll B \Leftrightarrow(\exists a \in A)(\exists b \in B)(a \ll b), \\
A \oplus B=\bigcup_{a \in A, b \in B} a \oplus b .
\end{gathered}
$$

We also define $0^{*}=1$ and $A^{*}=\left\{a^{*} \mid a \in A\right\}$. The notation $x \ll y$ and $A \ll B$ will be also written as $y \gg x$ and $B \gg A$, respectively.

Proposition 2.2. [2] Every hyper $M V$-algebra $M$ satisfies the following assertions:

(b1) $(A \oplus B) \oplus C=A \oplus(B \oplus C)$,

(b2) $0 \ll x, \quad x \ll 1$,

(b3) $x \ll x$,

(b4) $x \ll y \Rightarrow y^{*} \ll x^{*}$,

(b5) $A \ll B \Rightarrow B^{*} \ll A^{*}$,

(b6) $A \ll A$,

(b7) $A \subseteq B \Rightarrow A \ll B$,

(b8) $x \ll x \oplus y, \quad A \ll A \oplus B$,

(b9) $\left(A^{*}\right)^{*}=A$,

(b10) $0 \oplus 0=\{0\}$,

(b11) $x \in x \oplus 0$,

(b12) $y \in x \oplus 0 \Rightarrow y \ll x$,

(b13) $y \oplus 0=x \oplus 0 \Rightarrow x=y$

for all $x, y, z \in M$ and subsets $A, B$ and $C$ of $M$.

Definition 2.3. [2] A nonempty subset $S$ of a hyper MV-algebra $M$ is called a hyper $M V$-subalgebra of $M$ if $S$ is a hyper MV-algebra under the hyper operation " $\oplus$ " and the unary operation "*" on $M$.

LemMa 2.4. [2] A nonempty subset $S$ of a hyper $M V$-algebra $M$ is hyper $M V$-subalgebra of $M$ if and only if $x^{*} \in S$ and $x \oplus y \subseteq S$ for all $x, y \in S$.

Definition 2.5. [4] A nonempty subset $D$ of $M$ is called a weak hyper $M V$-deductive system of $M$ if it satisfies:

(c1) $0 \in D$,

(c2) $(\forall x, y \in M)\left(\left(x^{*} \oplus y\right)^{*} \subseteq D, y \in D \Rightarrow x \in D\right)$. 


\begin{tabular}{|c|c|c|c|c|c|c|}
\hline$\oplus$ & 0 & $a$ & $b$ & 1 & & $x^{*}$ \\
\hline 0 & $\{0\}$ & $\{0, a\}$ & $\{0, a, b\}$ & $\{0, a, b, 1\}$ & & 1 \\
\hline$a$ & $\{0, a\}$ & $\{0, a\}$ & $\{0, a, b, 1\}$ & $\{0, a, b, 1\}$ & & $b$ \\
\hline$b$ & $\{0, a, b\}$ & $\{0, a, b, 1\}$ & $\{0, a, b, 1\}$ & $\{0, a, b, 1\}$ & & $a$ \\
\hline 1 & $\{0, a, b, 1\}$ & $\{0, a, b, 1\}$ & $\{0, a, b, 1\}$ & $\{0, a, b, 1\}$ & & 0 \\
\hline
\end{tabular}

TABLE 1. $\oplus$-multiplication and unary operation

Definition 2.6. [4] A non-empty subset $D$ of $M$ is called a hyper $M V$-deductive system of $M$ if it satisfies (c1) and

(c3) $(\forall x, y \in M)\left(\left(x^{*} \oplus y\right)^{*} \ll D, y \in D \Rightarrow x \in D\right)$.

\section{Skeletons of hyper MV-algebras}

In what follows let $M$ denote a hyper MV-algebra unless otherwise specified.

Definition 3.1. A nonempty subset $K$ of a hyper MV-algebra $M$ is called a $(\subseteq, \in)$-skeleton of $M$ if it satisfies:

(d1) $1 \in K$,

(d2) $(\forall x \in K)(\forall y \in M)\left(x^{*} \oplus y \subseteq K \Rightarrow y \in K\right)$.

EXAMPLE 3.2. Let $M=\{0, a, b, 1\}$ be a set with the hyper operation " $\oplus$ " and the unary operation "*" which are given by Table 1 . Then $\left(M, \oplus,{ }^{*}, 0\right)$ is a hyper MV-algebra. It is easy to check that $K_{1}:=\{1, a\}$, $K_{2}:=\{1, b\}$ and $K_{3}:=\{1, a, b\}$ are $(\subseteq, \in)$-skeletons of $M$.

THeOREM 3.3. If $D$ is a weak hyper $M V$-deductive system of $M$, then the set

$$
D^{*}:=\left\{a^{*} \mid a \in D\right\}
$$

is a $(\subseteq, \in)$-skeleton of $M$.

Proof. Obviously, $1 \in D^{*}$. Let $x \in D^{*}$ and $y \in M$ be such that $x^{*} \oplus y \subseteq D^{*}$. Using (a2) and (a3), we have

$$
\left(\left(y^{*}\right)^{*} \oplus x^{*}\right)^{*}=\left(y \oplus x^{*}\right)^{*}=\left(x^{*} \oplus y\right)^{*} \subseteq D .
$$

It follows from (c2) that $y^{*} \in D$ so that $y \in D^{*}$. Hence $D^{*}$ is a $(\subseteq$, $\in)$-skeleton of $M$. 


\begin{tabular}{l|lllll|l}
\hline$\oplus$ & 0 & $a$ & $b$ & 1 & & $x^{*}$ \\
\hline 0 & $\{0\}$ & $\{0, a\}$ & $\{0, b\}$ & $\{0, a, b, 1\}$ & 0 & 1 \\
$a$ & $\{0, a\}$ & $\{a\}$ & $\{0, a, b, 1\}$ & $\{0, a, b, 1\}$ & $a$ & $b$ \\
$b$ & $\{0, b\}$ & $\{0, a, b, 1\}$ & $\{0, a, b, 1\}$ & $\{0, a, b, 1\}$ & $b$ & $a$ \\
1 & $\{0, a, b, 1\}$ & $\{0, a, b, 1\}$ & $\{0, a, b, 1\}$ & $\{0, a, b, 1\}$ & 1 & 0 \\
\hline
\end{tabular}

TABLE 2. $\oplus$-multiplication and unary operation

Theorem 3.4. Let $M$ be a hyper $M V$-algebra satisfying the following condition:

$$
(\forall x, y \in M)(y \in x \oplus y)
$$

Then every subset of $M$ containing 1 is a $(\subseteq, \in)$-skeleton of $M$.

Proof. Let $K$ be a subset of $M$ containing 1 , and let $x, y \in M$ be such that $x^{*} \oplus y \subseteq K$ and $x \in K$. Assume that $y \notin K$. Then $y \in x^{*} \oplus y$ by (3.1), and so $x^{*} \oplus y \nsubseteq K$. This is a contradiction, and therefore $y \in K$, proving that $K$ is a $(\subseteq, \in)$-skeleton of $M$.

Theorem 3.5. If $K_{1}$ and $K_{2}$ are $(\subseteq, \in)$-skeletons of a hyper $M V$ algebra $M$,then so is $K_{1} \cap K_{2}$.

Proof. Straightforward.

The following example shows that the union of $(\subseteq, \in)$-skeletons of a hyper MV-algebra $M$ is not a $(\subseteq, \in)$-skeletons of a hyper MV-algebra $M$.

Definition 3.6. A nonempty subset $K$ of a hyper MV-algebra $M$ is called a $(\gg, \in)$-skeleton of $M$ if it satisfies (d1) and

(d3) $(\forall x \in K)(\forall y \in M)\left(x^{*} \oplus y \gg K \Rightarrow y \in K\right)$.

EXAMPLE 3.7. Let $M=\{0, a, b, 1\}$ be a set with the hyper operation " $\oplus$ " and the unary operation "*" which are given by Table 2 . Then $\left(M, \oplus,{ }^{*}, 0\right)$ is a hyper MV-algebra. It is easy to check that $K_{2}:=\{1, b\}$ is a $(\gg, \in)$-skeleton of $M$, but $K_{1}:=\{1, a\}$ is not a $(\gg, \in)$-skeleton of $M$ since $a^{*} \oplus b=b \oplus b=\{0, a, b, 1\} \gg\{1, a\}$.

Proposition 3.8. Every ( $\gg, \in)$-skeleton $K$ of $M$ satisfies the following condition:

$$
(\forall x \in K)(\forall y \in M)(x \ll y \Rightarrow y \in K) .
$$

Proof. Let $x, y \in M$ be such that $x \ll y$ and $x \in K$. Then $1 \in x^{*} \oplus y$, and so $x^{*} \oplus y \gg K$ by (d1) and (b3). It follows from (d3) that $y \in K$. 
The following example shows that a $(\subseteq, \in)$-skeleton $K$ of $M$ may not satisfy the condition (3.2).

ExAMPle 3.9. Consider a hyper MV-algebra $M$ in Example 3.2. Then $K_{1}:=\{1, a\}$ is a $(\subseteq, \in)$-skeleton of $M$ which does not satisfy (3.2).

Let $M$ satisfy the condition (3.1) and let $K$ be a subset of $M$ containing 1. Then $K$ may not be a $(\gg, \in)$-skeleton of $M$ as seen in Example 3.7 .

Theorem 3.10. Every $(\gg, \in)$-skeleton is a $(\subseteq, \in)$-skeleton.

Proof. Let $K$ be a $(\gg, \in)$-skeleton of $M$. Assume that $x^{*} \oplus y \subseteq K$ for all $x \in K$ and $y \in M$. Then $a \ll a$ for all $a \in x^{*} \oplus y$, and so $x^{*} \oplus y \gg K$. Using (d3), we have $y \in K$. Hence $K$ is $(\subseteq, \in)$-skeleton of $M$.

THEOREM 3.11. If $D$ is a hyper $M V$-deductive system of $M$, then the set

$$
D^{*}:=\left\{a^{*} \mid a \in D\right\}
$$

is a $(\gg, \in)$-skeleton of $M$.

Proof. Obviously, $1 \in D^{*}$. Let $x \in D^{*}$ and $y \in M$ be such that $x^{*} \oplus y \gg D^{*}$. Then $\left(\left(y^{*}\right)^{*} \oplus x^{*}\right)^{*}=\left(x^{*} \oplus y\right)^{*} \ll D$ by (a2), (a3), (b5) and (b9). It follows from (c3) that $y^{*} \in D$ so from (a3) that $y \in D^{*}$. Hence $D^{*}$ is a $(\gg, \in)$-skeleton of $M$.

THEOREM 3.12. If $K_{1}$ and $K_{2}$ are $(\gg, \in)$-skeletons of a hyper $M V$ algebra $M$, then so is $K_{1} \cap K_{2}$.

Proof. Straightforward.

The following example shows that the union of $(\gg, \in)$-skeletons of a hyper MV-algebra $M$ is not a $(\gg, \in)$-skeletons of a hyper MV-algebra $M$.

Definition 3.13. A nonempty subset $K$ of a hyper MV-algebra $M$ is called a $\left(\ll^{*}, \in\right)$-skeleton of $M$ if it satisfies (d1) and (d4) $(\forall x \in K)(\forall y \in M \backslash\{0\})\left(x^{*} \oplus y \ll K^{*} \Rightarrow y \in K\right)$.

EXAMPLE 3.14. For a hyper MV-algebra $M$, the set $K:=M \backslash\{0\}$ is a $\left(\ll^{*}, \in\right)$-skeleton of $M$.

EXAMPLE 3.15. Let $M=\{0, b, 1\}$ be a set with the hyper operation " $\oplus$ " and the unary operation "*" which are given by Table 3 . Then $\left(M, \oplus,{ }^{*}, 0\right)$ is a hyper MV-algebra. It is easy to check that $K_{1}:=\{1\}$ and $K_{2}:=\{1, b\}$ are $\left(\ll^{*}, \in\right)$-skeletons of $M$. But $K:=\{1,0\}$ is not a $\left(\ll^{*}, \in\right)$-skeleton of $M$ since $1^{*} \oplus b=\{b\} \ll K^{*}$ and $b \notin K^{*}$. 


\begin{tabular}{l|lllll|l}
\hline$\oplus$ & 0 & $b$ & 1 & & $x$ & $x^{*}$ \\
\hline 0 & $\{0\}$ & $\{b\}$ & $\{1\}$ & & 1 \\
$b$ & $\{b\}$ & $\{0, b, 1\}$ & $\{0, b, 1\}$ & $b$ & $b$ \\
1 & $\{1\}$ & $\{0, b, 1\}$ & $\{1\}$ & & 0
\end{tabular}

TABLE 3. $\oplus$-multiplication and unary operation

REMARK 3.16. Let $K$ be a proper subset of a hyper MV-algebra $M$. Then there exists $y \in M \backslash K$. If $K$ contains 0 , then $1 \in K^{*}$ and so $x^{*} \oplus y \ll K^{*}$ for all $x \in K$. This shows that every $\left(\ll^{*}, \in\right)$-skeleton does not contain 0 .

REMARK 3.17. There is no relation between a $(\subseteq, \in)$-skeleton and a $\left(\ll^{*}, \in\right)$-skeleton. In fact, $K_{1}:=\{1, a\}$ in Example 3.2 is a $(\subseteq, \in)$ skeleton of $M$. But it is not a $\left(\ll^{*}, \in\right)$-skeleton of $M$. Also, $K_{2}:=\{1, b\}$ in Example 3.15 is a $\left(\ll^{*}, \in\right)$-skeleton of $M$ which is not a $(\subseteq, \in)$-skeleton of $M$.

REMARK 3.18. A (», $\in)$-skeleton may not be a $\left(\ll^{*}, \in\right)$-skeleton. For example, $K_{1}:=\{1, b\}$ in Example 3.7 is a $(\gg, \in)$-skeleton of $M$ which is not a $\left(\ll^{*}, \in\right)$-skeleton of $M$.

THEOREM 3.19. If $K_{1}$ and $K_{2}$ are $\left(\ll^{*}, \in\right)$-skeletons of a hyper $M V$-algebra $M$, then so is $K_{1} \cap K_{2}$.

Proof. Straightforward.

The following example shows that the union of $\left(\ll^{*}, \in\right)$-skeletons of a hyper MV-algebra $M$ is not a $\left(\ll^{*}, \in\right)$-skeletons of a hyper MV-algebra $M$.

Definition 3.20. A nonempty subset $K$ of a hyper MV-algebra $M$ is called a hyper $M V$-skeleton of $M$ if it satisfies

(d5) $(\forall x \in K)(\forall y \in M)\left(x^{*} \oplus y \ll K \Rightarrow y \in K\right)$.

EXAMPLE 3.21. Let $M=\{0, a, b, 1\}$ be a set with the hyper operation " $\oplus$ " and the unary operation "*" which are given by Table 4 . Then $\left(M, \oplus,{ }^{*}, 0\right)$ is a hyper MV-algebra. It is easy to check that $K_{a}:=\{a\}$ is a hyper MV-skeleton of $M$. But $K_{b}:=\{b\}$ is not a hyper MV-skeleton of $M$ since $b^{*} \oplus a=a \oplus a=\{0, a\} \ll\{b\}=K_{b}$ and $a \notin K_{b}$.

Definition 3.22. A nonempty subset $K$ of a hyper MV-algebra $M$ is called a weak hyper $M V$-skeleton of $M$ if it satisfies 


\begin{tabular}{l|lllllll}
\hline$\oplus$ & 0 & $a$ & $b$ & 1 & & $x$ & $x^{*}$ \\
\hline 0 & $\{0\}$ & $\{0, a\}$ & $\{b\}$ & $\{b, 1\}$ & & 1 \\
$a$ & $\{0, a\}$ & $\{0, a\}$ & $\{b, 1\}$ & $\{b, 1\}$ & $a$ & $b$ \\
$b$ & $\{b\}$ & $\{b, 1\}$ & $\{b, 1\}$ & $\{b, 1\}$ & $b$ & $a$ \\
1 & $\{b, 1\}$ & $\{b, 1\}$ & $\{b, 1\}$ & $\{b, 1\}$ & 1 & 0 \\
\hline
\end{tabular}

TABLE 4. $\oplus$-multiplication and unary operation

(d6) $(\forall x \in K)(\forall y \in M)\left(\left(x^{*} \oplus y\right) \cap K \neq \emptyset \Rightarrow y \in K\right)$.

Lemma 3.23. For every subset $K$ of a hyper $M V$-algebra $M$, we have

$$
(\forall x, y \in M)\left(\left(x^{*} \oplus y\right) \cap K \neq \emptyset \Rightarrow x^{*} \oplus y \ll K\right) .
$$

Proof. Straightforward.

Theorem 3.24. Every hyper $M V$-skeleton is a weak hyper $M V$ skeleton.

Proof. It follows immediately from Lemma 3.23.

The following example shows that the converse of Theorem 3.24 may not be true.

ExAMPle 3.25. Consider a hyper MV-algebra $M$ which is given in Example 3.21. Then $K:=\{b, 1\}$ is a weak hyper MV-skeleton of $M$, but it is not a hyper MV-skeleton of $M$ since $b^{*} \oplus a=\{0, a\} \ll K$ and $a \notin K$.

THEOREM 3.26. If $K_{1}$ and $K_{2}$ are (weak) hyper $M V$-skeletons of a hyper $M V$-algebra $M$, then so is $K_{1} \cap K_{2}$.

Proof. Straightforward.

The following example shows that the union of (weak) hyper MVskeletons of a hyper MV-algebra $M$ is not a (weak) hyper MV-skeleton of a hyper MV-algebra $M$.

Definition 3.27. A nonempty subset $K$ of a hyper MV-algebra $M$ is called a strong $\left(\subseteq^{*}, \in\right)$-skeleton of $M$ if it satisfies (d1) and

(d7) $(\forall x \in K)(\forall y \in M)\left(x^{*} \oplus y \subseteq K^{*} \Rightarrow y \in K\right)$. 


\begin{tabular}{l|lllll|l}
\hline$\oplus$ & 0 & $a$ & $b$ & 1 & & $x^{*}$ \\
\hline 0 & $\{0\}$ & $\{0, a, b\}$ & $\{0, b\}$ & $\{0, a, b, 1\}$ & 0 & 1 \\
$a$ & $\{0, a, b\}$ & $\{0,1\}$ & $\{0, a, b, 1\}$ & $\{0, a, b, 1\}$ & $a$ & $b$ \\
$b$ & $\{0, b\}$ & $\{0, a, b, 1\}$ & $\{b\}$ & $\{0, a, b, 1\}$ & $b$ & $a$ \\
1 & $\{0, a, b, 1\}$ & $\{0, a, b, 1\}$ & $\{0, a, b, 1\}$ & $\{0, a, b, 1\}$ & 1 & 0 \\
\hline
\end{tabular}

TABLE 5. $\oplus$-multiplication and unary operation

EXAMPLE 3.28. Let $M=\{0, a, b, 1\}$ be a set with the hyper operation " $\oplus$ " and the unary operation " $*$ " which are given by Table 5 . Then $\left(M, \oplus,{ }^{*}, 0\right)$ is a hyper MV-algebra. It is easy to check that $K_{1}:=\{0,1\}$ is a strong $\left(\subseteq^{*}, \in\right)$-skeleton of $M$, but $K_{2}:=\{1\}$ is not a strong $\left(\subseteq^{*}\right.$, $\in)$-skeleton of $M$ since $1^{*} \oplus 0=\{0\} \subseteq K_{2}^{*}$ and $0 \notin K_{2}$.

Definition 3.29. A nonempty subset $K$ of a hyper MV-algebra $M$ is called a $\left(\subseteq^{*}, \in\right)$-skeleton of $M$ if it satisfies (d1) and

(d8) $(\forall x \in K)(\forall y \in M \backslash\{0\})\left(x^{*} \oplus y \subseteq K^{*} \Rightarrow y \in K\right)$.

EXAMPLE 3.30. Let $\left(M, \oplus,{ }^{*}, 0\right)$ be a hyper MV-algebra which is given in Example 3.28. Then $K:=\{a, b, 1\}$ is a $\left(\subseteq^{*}, \in\right)$-skeleton of $M$. But $K_{a}:=\{a, 1\}$ is not a $\left(\subseteq^{*}, \in\right)$-skeleton of $M$ since $a^{*} \oplus b=\{b\} \subseteq K_{a}^{*}$ but $b \notin K_{a}$.

Obviously, if $M$ is a hyper $\mathrm{MV}$-algebra, then $M \backslash\{0\}$ is a $\left(\subseteq^{*}, \in\right)$ skeleton of $M$. Note that every strong $\left(\subseteq^{*}, \in\right)$-skeleton is a $\left(\subseteq^{*}, \in\right)$ skeleton, but the converse is not true as seen in the following example.

EXAMPLE 3.31. Let $\left(M, \oplus,{ }^{*}, 0\right)$ be a hyper MV-algebra which is given in Example 3.28. Then $K:=\{a, b, 1\}$ is a $\left(\subseteq^{*}, \in\right)$-skeleton of $M$, but it is not a strong $\left(\subseteq^{*}, \in\right)$-skeleton of $M$ since $a^{*} \oplus 0=\{0, b\} \subseteq K^{*}$ but $0 \notin K$.

Using $(\mathrm{d} 7)$, we know that every $\left(\ll^{*}, \in\right)$-skeleton is a $\left(\subseteq^{*}, \in\right)$-skeleton. But the converse may not hold as seen in the following example.

EXAMPLE 3.32. Let $\left(M, \oplus,{ }^{*}, 0\right)$ be a hyper MV-algebra which is given in Example 3.28. Then $K:=\{a, b, 1\}$ is a $\left(\subseteq^{*}, \in\right)$-skeleton of $M$. But it is not a $\left(\ll^{*}, \in\right)$-skeleton of $M$ since $a^{*} \oplus 0=\{0, b\} \ll K^{*}$ but $0 \notin K$.

THEOREM 3.33. If $K_{1}$ and $K_{2}$ are (strong) $\left(\subseteq^{*}, \in\right)$-skeletons of a hyper $M V$-algebra $M$, then so is $K_{1} \cap K_{2}$.

Proof. Straightforward. 
The following example shows that the union of (strong) $\left(\subseteq^{*}, \in\right)$ skeletons of a hyper MV-algebra $M$ is not a (strong) $\left(\subseteq^{*}, \in\right)$-skeletons of $M$.

REMARK 3.34. There is no relation between a $(\gg, \in)$-skeleton and a strong $\left(\subseteq^{*}, \in\right)$-skeleton as shown by the following examples.

EXAMPLE 3.35. Let $\left(M, \oplus,{ }^{*}, 0\right)$ be a hyper MV-algebra which is given in Example 3.28. Then $K:=\{0,1\}$ is a strong $\left(\subseteq^{*}, \in\right)$-skeleton of $M$, but not a $(\gg, \in)$-skeleton of $M$ since $1^{*} \oplus b=\{0, b\} \gg K$ but $b \notin K$.

EXAMPLE 3.36. Let $M$ be a hyper MV-algebra which is given in Example 3.7. Then $K:=\{1, b\}$ is a $(\gg, \in)$-skeleton of $M$, but not a strong $\left(\subseteq^{*}, \in\right)$-skeleton of $M$ since $b^{*} \oplus 0=\{0, a\} \subseteq K^{*}$, but $0 \notin K$.

REMARK 3.37. There is no relation between a $\left(\ll^{*}, \in\right)$-skeleton and a strong $\left(\subseteq^{*}, \in\right)$-skeleton as shown by the following examples.

EXAMPLE 3.38. Let $\left(M, \oplus,{ }^{*}, 0\right)$ be a hyper MV-algebra which is given in Example 3.28. Then $K:=\{0,1\}$ is a strong $\left(\subseteq^{*}, \in\right)$-skeleton of $M$, but not a $\left(\ll^{*}, \in\right)$-skeleton of $M$ since $0^{*} \oplus a=\{0, a, b, 1\} \ll K^{*}$ but $a \notin K$.

\section{References}

[1] C.C. Change, Algebraic analysis of many valued logic, Transactions American Mathematical Society, 88 (1958), 476-490.

[2] Sh. Ghorbani, On hyper MV-algebras, Ph. D. thesis, Shahbid Bahonar University of Kerman, 2007.

[3] Sh. Ghorbani, A. Hasankhani and E. Eslami, Hyper $M V$-algerbas, Set-Valued Math. Appl. (to appear).

[4] Y. B. Jun, M. S. Kang and H. S. Kim, Hyper $M V$-deductive systems of hyper $M V$-algebras, Internat. J. Fuzzy Systems (submitted).

[5] F. Marty, Sur une generalization de la notion de groups, 8th congress Math. Scandinaves, Stockholm, (1934), 45-49.

[6] L. Torkzadeh and A. Ahadpanah, Hyper MV-ideals in hyper MV-algerbas, Math. Logic. Quaterly (submitted).

Min Su Kang

Department of Mathematics

Hanyang University

Seoul 133-791, Korea

e-mail : sinchangmyun@hanmail.net 